\title{
A new finite-element shell model for arterial growth and remodeling after stent implantation
}

\author{
Joan D. Laubrie, S. Jamaleddin Mousavi, Stéphane Avril \\ Mines Saint-Étienne, Univ Lyon, Univ Jean Monnet, INSERM, U 1059 Sainbiose, Centre CIS, F - 42023 \\ Saint-Étienne France.
}

\begin{abstract}
The goal of this paper is to study computationally how blood vessels adapt when they are exposed to a mechanobiological insult, namely a sudden change of their biomechanical conditions such as proteolytic injuries or implantation. Adaptation occurs through growth and remodeling $(\mathrm{G} \& \mathrm{R})$, consisting in mass production or removal of structural proteins, such as collagen, until restoring the initial homeostatic biomechanical conditions. In some circumstances, the initial conditions can never be recovered and arteries evolve towards unstable pathological conditions, such as aneurysms, which are responsible for significant morbidity and mortality. Therefore, computational predictions of G\&R under different circumstances can be helpful in understanding fundamentally how arterial pathologies progress. For that we have developed a low-cost open-source finite-element 2D axisymmetric shell model (FEM) of the arterial wall. The constitutive equations for static equilibrium used to model the stress-strain behavior and the G\&R response are expressed within the homogenized constrained mixture theory. The originality is to integrate the layer-specific behavior of both arterial layers (media and adventitia) into the model. Considering different mechanobiological insults, our results show that the resulting arterial dilatation is strongly correlated with the media thickness. The adaptation to stent implantation is particularly interesting. For large stent over-sizing ratios, the artery cannot recover from the mechanobiological insult and dilates forever, whereas dilatation stabilizes after a transient period for more moderate oversizing ratios. We also show that stent implantation induces a different response in an aneurysm or in a healthy artery, the latter yielding more unstable G\&R. Finally, our G\&R model can efficiently predict, with very low computational cost, fundamental aspects of arterial adaptation induced by clinical procedures.
\end{abstract}

\section{List of symbols}

In following $j \in\left\{e, c_{i}, m\right\}$ and $k \in\left\{c_{i}, m\right\}$

$\boldsymbol{a}_{0}^{k} \quad$ The unit vector pointing direction of the $k$ th fibre

$B^{j} \quad$ Growth direction tensor of $j$ th constituent

$C^{e}$ Stress-like material parameter of elastin in neo-Hookean energy

$C_{1}^{k} \quad$ Stress-like material parameter of $k$ th fibre in Fung-type energy

$C_{2}^{k} \quad$ Dimensionless material parameter of $k$ th fibre in Fung-type energy 


\begin{tabular}{|c|c|}
\hline$C_{e}^{j}$ & Elastic right Cauchy-Green deformation tensor of the $j$ th constituent \\
\hline $\mathbb{C}$ & Cauchy-Green deformation tensor of the mixture \\
\hline $\mathbb{C}^{e}$ & Second Piola-Kirchhoff stress tensor of elastin \\
\hline $\mathbb{C}^{c_{i}}$ & Second Piola-Kirchhoff stress tensor of $i$ th collagen \\
\hline $\mathbb{C}_{\text {pas }}^{m}$ & Second Piola-Kirchhoff passive stress tensor of smooth muscle cells \\
\hline $\mathbb{C}_{a c t}^{m}$ & Second Piola-Kirchhoff active stress tensor of smooth muscle cells \\
\hline$d$ & Vector of cross section direction of the shell in current coordinates on the mid-surface \\
\hline$D_{\max }$ & Maximum damage \\
\hline$\dot{D}^{j}$ & Any additional mass deposition or degradation in $j$ th constituent \\
\hline$D \delta W_{\text {int }}$ & Linearized internal virtual work \\
\hline$E$ & Green-Lagrange strain tensor of the mixture \\
\hline$E_{z r}$ & Green-Lagrange shear strain of the mixture between $Z$ and $R$ faces \\
\hline$F$ & Total deformation gradient tensor of the mixture \\
\hline$F_{g}$ & Total growth deformation gradient tensor of the mixture \\
\hline $\boldsymbol{F}_{e}^{j}$ & Elastic deformation gradient tensor of the $j$ th constituent \\
\hline $\boldsymbol{F}_{g r}^{j}$ & Inelastic deformation gradient tensor of the $j$ th constituent \\
\hline $\boldsymbol{F}_{g}^{j}$ & Growth deformation gradient tensor of the $j$ th constituent \\
\hline $\boldsymbol{F}_{r}^{j}$ & Remodeling deformation gradient tensor of the $j$ th constituent \\
\hline$G_{h z}^{e}$ & Axial deposition stretch of elastin \\
\hline$G_{h \theta}^{e}$ & Circumferential depostion stretch of elastin \\
\hline$G_{h r}^{e}$ & Radial or thickness deposition stretch of elastin \\
\hline$G_{h}^{k}$ & Fibre deposition stretch of $k$ th fibre \\
\hline$\dot{G}$ & Growth-related deformation gradient rate tensor of the mixture \\
\hline$\dot{G}^{j}$ & Growth-related deformation gradient rate tensor of $j$ th constituent \\
\hline$h$ & Temporal wall thickness \\
\hline$h_{0}$ & Initial wall thickness \\
\hline$h^{*}$ & Thickness at the interface between the media and the adventitia \\
\hline$k_{\sigma}^{j}$ & Gain parameter of $j$ th constituent \\
\hline$L_{s}$ & Stent length \\
\hline$L_{d a m}$ & Spacial damage spread \\
\hline $\boldsymbol{L}_{r}^{j}$ & Remodeling velocity gradient tensor of the $j$ th constituent \\
\hline$N$ & Vector on cross section direction of the shell in material coordinates on the mid-surface \\
\hline$\hat{p}$ & Luminal reference pressure \\
\hline$R$ & Material radial coordinate of shell element \\
\hline$S_{a c t m a x}$ & Maximal active Cauchy stress \\
\hline$S$ & Second Piola-Kirchhoff stress tensor \\
\hline$S^{e}$ & Second Piola-Kirchhoff stress tensor of elastin \\
\hline$S^{c_{i}}$ & Second Piola-Kirchhoff stress tensor of $i$ th collagen \\
\hline$S_{\text {pas }}^{m}$ & Second Piola-Kirchhoff passive stress tensor of smooth muscle cells \\
\hline$S_{a c t}^{m}$ & Second Piola-Kirchhoff active stress tensor of smooth muscle cells \\
\hline$S_{p r e}^{j}$ & Homeostatic second Piola-Kirchhoff stress tensor of $j$ th constituent \\
\hline$S_{t}^{j}$ & Temporal second Piola-Kirchhoff stress tensor of $j$ th constituent \\
\hline$t_{\text {dam }}$ & Temporal damage spread \\
\hline
\end{tabular}




\begin{tabular}{|c|c|}
\hline$T^{j}$ & Half-life of $j$ th constituent \\
\hline$u$ & Displacement parallel to the shell \\
\hline$u$ & Displacement vector \\
\hline$w$ & Displacement normal to the shell \\
\hline$W_{M}$ & Strain energy function per unit reference volume in Media \\
\hline$W_{A}$ & Strain energy function per unit reference volume in Adventitia \\
\hline$Z$ & Material axial coordinate of shell \\
\hline$x$ & Position of a particle in the current configuration \\
\hline $\boldsymbol{x}_{M}$ & Position of a particle at mid-surface in the current configuration \\
\hline$X$ & Position of a particle in material configuration \\
\hline $\boldsymbol{X}_{M}$ & Position of a particle at mid-surface in the reference material configuration \\
\hline$\alpha^{k}$ & The orientation of the $k$ th fibre \\
\hline$\beta$ & Bending angle of the shell \\
\hline$\delta W_{\text {int }}$ & Internal virtual work \\
\hline$\epsilon$ & Penalty of the shell element method \\
\hline$\theta$ & Orientation of the shell \\
\hline$\lambda_{z}$ & Total axial stretch \\
\hline$\lambda_{\theta}$ & Total circumferential stretch \\
\hline$\lambda_{r}$ & Total radial or thickness stretch \\
\hline$\lambda_{z}^{e}$ & Elastic axial stretch of elastin \\
\hline$\lambda_{\theta}^{e}$ & Elastic circumferential stretch of elastin \\
\hline$\lambda_{r}^{e}$ & Elastic radial or thickness stretch of elastin \\
\hline$\lambda^{k}$ & Total stretch of the $k$ th fibre in its direction \\
\hline$\lambda_{e}^{k}$ & Elastic stretch of the $k$ th fibre in its direction \\
\hline$\lambda_{r}^{k}$ & Remodeling stretch of the $k$ fibre in direction \\
\hline$\lambda_{\text {actmax }}$ & Active stretch of the fibre \\
\hline$\lambda_{0}^{m}$ & Zero active stretch of the fibre \\
\hline$\lambda_{\max }^{m}$ & Maximum active stretch of the fibre \\
\hline$\xi$ & Thickness parametric coordinate \\
\hline$\rho_{0}$ & Total mass density of the mixture (arterial wall) at $t=0$ \\
\hline$\rho_{t}$ & Total mass density of the mixture (arterial wall) \\
\hline$\rho_{t}^{j}$ & Mass density of the $j$ th constituent \\
\hline$\dot{\rho}_{t}^{j}$ & Mass turnover of the $j$ th constituent \\
\hline$\sigma_{t}^{j}$ & Temporal Cauchy stress of the $j$ th constituent \\
\hline$\sigma_{\text {pre }}^{j}$ & Homeostatic Cauchy stress of the $j$ th constituent \\
\hline$\phi_{M}^{e}$ & Mass fraction of the elastin in the media \\
\hline$\phi_{M}^{k}$ & Mass fraction of the $k$ th fibre in the media \\
\hline$\phi_{A}^{e}$ & Mass fraction of the elastin in the adventitia \\
\hline$\phi_{A}^{k}$ & Mass fraction of $k$ th fibre in the adventitia \\
\hline$\Psi^{e}$ & Strain energy function per unit reference mass of elastin \\
\hline$\Psi^{c_{i}}$ & Strain energy function per unit reference mass of $i$ th collagen fibre \\
\hline$\Psi_{\text {pas }}^{m}$ & Passive strain energy function per unit reference mass of smooth muscle cells \\
\hline$\Psi_{a c t}^{m}$ & Active strain energy function per unit reference mass of smooth muscle cells \\
\hline$\Omega^{r}$ & Reference configuration \\
\hline
\end{tabular}




\section{Introduction}

Vascular tissues, as other biological tissues, commonly maintain homeostatic conditions during routine function and therefore, they continually adapt to any mechanical and biochemical alteration in their surrounding. Any factor disturbing the preferred homeostatic state of arterial wall, such as permanent hypertension or disruption of elastin fibers [1], may induce vascular growth and remodeling $(G \& R)$ which is a vital process to maintain vessel function. At the tissue scale, this manifests through continuous mass changes of the existent components in the extracellular matrix (ECM) such as collagen, elastin and proteoglycans [2], [3]. When the arterial wall is unable to recover its homeostatic conditions through G\&R, arterial dysfunction may arise and end up with, for instance, an aneurysm, which is a permanent, degenerative and localized expansion of the arterial diameter. An aneurysm can lead to a wall dissection and rupture and potentially be a life-threatening condition.

In the past two decades, different computational approaches were developed to model G\&R of load-bearing soft tissues. These methods can be grouped in two major mathematical theories: a constrained mixture theory (CMT) determining the rates of mass removal and production of individual constituents within stressed configurations or a kinematic theory specifying an evolution equation for the stress-free configuration of the tissue as a whole. Although the latter is popular and conceptually more simple, it relies largely on heuristic definitions of growth [2]. Kinematic growth theories commonly split multiplicatively the total deformation gradient into elastic and inelastic parts, where the inelastic one is related to growth [4]. This theory has been widely used for single-constituent solid continuum [5], [6] as well as for homogenized [2], [7] and non-homogenized [8]-[11] constrained mixture models (CMMs). For example, Valentín et al [11], [12 modelled arterial wall adaptation and maladaptation to different cases, such as loss of smooth muscle cells (SMCs), elastin degradation and changes in fiber orientations and quantities. Watton et al [13], [14] quantified the interaction between collagen microstructure and mechanical stretch to model the growth of an abdominal aortic aneurysm (AAA). They introduced variables for the recruitment of collagen fibers to account for microstructural changes leading to the formation of an aneurysm. Watton et al [13] introduced the first bi-layered G\&R approach using a membrane model. Cyron et al 15 employed the CMT to capture G\&R of soft tissues due to altered mechanobiological stimuli. Moreover, Wilson et al [16] investigated the effects of collagen turnover and elastin loss on the formation of AAAs in a parametric study. Their results showed that a number of variables play a substantial role on radial dilatation and axial expansion of AAAs, including wall thickness, fiber stretch, maximum wall stress and evolving material properties.

Despite extensive endeavor to establish G\&R models, significant efforts are still needed to develop reliable models of aneurysm evolution. Moreover, adaptation after endovascular aneurysm repair has never been modelled so far. Therefore, there is still an important potential for $\mathrm{G} \& \mathrm{R}$ models to understand the adaptation of arteries before or after different clinical treatments.

In this work, our contribution was twofold. First, it was computational: we implemented an original layer-specific homogenized CMT-based finite element (FE) shell model to study transient G\&R effects of different clinically-relevant cases related to aneurysm growth and 
repair. Second it was physiological: we predicted the adaptation of an artery after stent implantation and showed the major effect of oversizing on the post-surgery outcomes.

\section{Material and methods}

\subsection{Strain Energy Function}

The homogenized CMT was employed as an hybrid approach to consider G\&R in the arterial wall [3]. Let us assume a material point of a mixture in the $\Omega^{\mathrm{r}}$ domain, represented by its position vector $\mathbf{X}$ in the reference configuration and by its position vector $\mathbf{x}=\chi(\mathbf{X})$ in the deformed configuration. The total deformation gradient tensor can be defined by

$$
\boldsymbol{F}=\nabla \boldsymbol{x}
$$

where $\nabla$ is the gradient operator. For a bi-layered shell element without any shear in cylindrical coordinates the total deformation gradient can be written as $\mathbf{F}=\operatorname{diag}\left[\lambda_{z} \lambda_{\theta} \lambda_{r}\right]$. Noting that $\lambda_{z}, \lambda_{\theta}$ and $\lambda_{r}$ are the total stretches in axial, circumferential and radial directions, respectively. Based on the CMT, we split the strain energy function of the wall, $W$, into contributions of elastin, collagen fiber families and SMCs. We assumed that SMCs are present only in the media layer and are aligned along the circumferential direction. They have both an active, $(\bullet)_{\text {act }}$, and a passive, $(\bullet)_{\text {pas }}$, behavior. We modeled the collagen by four fiber families aligned in circumferential, longitudinal and two diagonal directions respectively. We assumed the same strain energy functions for each constituent in the media and in the adventitia while their mass fraction were different in each layer and even between each collagen fiber family. Assuming $\Psi^{j}, j \in\left\{e, m, c_{i}\right\}$, is the strain energy density function of each constituent, the total strain energy density function in the media, $W_{M}$, and in the adventitia, $W_{A}$, layers were respectively calculated by

$$
\begin{aligned}
& W_{M}=\rho_{t}\left(\phi_{M}^{e} \Psi^{e}+\phi_{M}^{m}\left(\Psi_{\text {pas }}^{m}+\Psi_{a c t}^{m}\right)+\sum_{i=1}^{4} \phi_{M}^{c_{i}} \Psi^{c_{i}}\right) \\
& W_{A}=\rho_{t}\left(\phi_{A}^{e} \Psi^{e}+\phi_{A}^{m}\left(\Psi_{\text {pas }}^{m}+\Psi_{a c t}^{m}\right)+\sum_{i=1}^{4} \phi_{A}^{c_{i}} \Psi^{c_{i}}\right)
\end{aligned}
$$

where $\rho_{t}$ is the total density and $\phi_{M}^{j}$ and $\phi_{A}^{j}$ denote mass fractions of each $j$ th constituent in the media and in the adventitia, respectively. For each component, we assumed a strain energy density function to represent the corresponding hyperelastic behavior. We modeled the elastin behavior with a Neo-Hookean hyperelastic model as in [17], [18

$$
\Psi^{e}=\frac{C^{e}}{2}\left(\lambda_{z}^{e 2}+\lambda_{\theta}^{e 2}+\lambda_{r}^{e 2}-3\right)
$$

where $C^{e}$ is a stress-like material parameter while $\lambda_{z}^{e}, \lambda_{\theta}^{e}$ and $\lambda_{r}^{e}$ are stretches of the elastin component in axial, circumferential and radial directions, respectively. In the CMT, these 
stretches are calculated by considering the elastin deposition stretch, $G_{h}^{e}=\operatorname{diag}\left[G_{h z}^{e} G_{h \theta}^{e} G_{h r}^{e}\right]$, such as 17, 18

$$
\lambda_{z}^{e}=G_{h z}^{e} \lambda_{z}, \lambda_{\theta}^{e}=G_{h \theta}^{e} \lambda_{\theta}, \lambda_{r}^{e}=G_{h r}^{e} \lambda_{r}
$$

we modeled the Collagen fiber families by an anisotropic Fung-type exponential function such as

$$
\Psi^{c_{i}}=\frac{C_{1}^{c_{i}}}{4 C_{2}^{c_{i}}}\left(\exp \left(C_{2}^{c_{i}}\left(\lambda_{e}^{c_{i} 2}-1\right)^{2}\right)-1\right)
$$

where $C_{1}^{c_{i}}$ and $C_{2}^{c_{i}}$ are a stress-like and dimensionless material parameters, respectively, while $\lambda_{e}^{c_{i}}$ is the elastic contribution of the collagen fiber stretch obtained as

$$
\lambda_{e}^{c_{i}}=\frac{\lambda^{c_{i}}}{\lambda_{r}^{c_{i}}} \text { with } \lambda^{c_{i}}=G_{h}^{c_{i}} \sqrt{\lambda_{z}^{2} \cos ^{2} \alpha^{c_{i}}+\lambda_{\theta}^{2} \sin ^{2} \alpha^{c_{i}}}
$$

where $\lambda^{c_{i}}, \lambda_{r}^{c_{i}}, G_{h}^{c_{i}}$ and $\alpha^{c_{i}}$ are the total stretch, remodeling stretch (cf. 13), deposition stretches and orientation angles of the different collagen fiber families, respectively. We also modeled the passive behavior of SMCs by an anisotropic Fung-type exponential function such as

$$
\Psi_{\text {pas }}^{m}=\frac{C_{1}^{m}}{4 C_{2}^{m}}\left(\exp \left(C_{2}^{m}\left(\lambda_{e}^{m 2}-1\right)^{2}\right)-1\right)
$$

while we modeled its active behavior according to Braeu et al [7],

$$
\Psi_{a c t}^{m}=\frac{\sigma_{a c t \max }}{\rho_{0}}\left(\lambda_{a c t}+\frac{\left(\lambda_{\max }^{m}-\lambda_{a c t}\right)^{3}}{3\left(\lambda_{\max }^{m}-\lambda_{0}^{m}\right)^{2}}\right)
$$

where $C_{1}^{m}$ and $C_{2}^{m}$ are stress-like and dimension-less material parameters, respectively, $\sigma_{\text {actmax }}$ is the maximal active Cauchy stress, $\lambda_{a c t}$ is the active stretch in the fiber direction, $\lambda_{0}^{m}$ and $\lambda_{\max }^{m}$ are the zero and maximum active stretches and $\rho_{0}$ denotes the total mixture density in the reference configuration; $\lambda_{e}^{m}$ is the elastic contribution of SMCs calculated such as

$$
\lambda_{e}^{m}=\frac{\lambda^{m}}{\lambda_{r}^{m}} \text { with } \lambda^{m}=G_{h}^{m} \sqrt{\lambda_{z}^{2} \cos ^{2} \alpha^{m}+\lambda_{\theta}^{2} \sin ^{2} \alpha^{m}}
$$

where $\lambda^{m}, \lambda_{r}^{m}$ and $G_{h}^{m}$ are the total stretch, remodeling stretch (cf. 13), deposition stretch and orientation angle of SMCs, respectively.

Given the strain energy function, the second Piola-Kirchoff stress tensor and the fourth order elasticity tensor of the mixture were obtained as

$$
\begin{aligned}
& S=2 \frac{\partial W}{\partial \boldsymbol{C}}=S^{e}+S_{\text {pas }}^{m}+S_{\text {act }}^{m}+\sum_{i=1}^{4} \boldsymbol{S}^{c_{i}} \\
& \mathbb{C}=2 \frac{\partial S}{\partial \boldsymbol{C}}=\mathbb{C}^{e}+\mathbb{C}_{\text {pas }}^{m}+\mathbb{C}_{\text {act }}^{m}+\sum_{i=1}^{4} \mathbb{C}^{c_{i}}
\end{aligned}
$$

where we included the penalty term of incompressibility into the expressions of stress and elasticity tensor of elastin. We considered a bi-layer arterial wall assuming that the media is mainly occupied by elastin and SMCs while the adventitia is mainly composed of collagen fibers. 


\subsection{Growth and remodeling}

Similarly to classical G\&R studies [2], [4], we split the total deformation gradient of each constituent into an elastic $\boldsymbol{F}_{e}^{j}$ and an inelastic $\boldsymbol{F}_{g r}^{j}$ contribution such as

$$
\boldsymbol{F}^{j}=\boldsymbol{F}_{e}^{j} \boldsymbol{F}_{g r}^{j}
$$

The idea of the homogenized CMT is to pool all the sequential mass additions within one single constituent using temporal homogenization. To do so, three assumptions are made: (i) the mechanical properties are changed by G\&R, (ii) survival mass (mass turnover) functions are exponential and (iii) inelastic deformations, $\boldsymbol{F}_{g r}^{j}=\boldsymbol{F}_{g}^{j} \boldsymbol{F}_{r}^{j}$, are in turn decomposed into growthrelated, $\boldsymbol{F}_{g}^{j}$, and remodeling-related (turnover-related), $\boldsymbol{F}_{r}^{j}$, contributions. In this model, a single average inelastic deformation gradient $\boldsymbol{F}_{g r}^{j}$ is defined. The growth-based part captures the changes of the differential volume element due to mass variations. The model can handle isotropic or anisotropic growth, the latter being more relevant for arteries and manifesting with thickening or thinning effects [19].

We assumed that $G \& R$ is a stress mediated process which tends to minimize deviations between the current stresses and a reference stress metrics named homeostatic stress. Therefore, the rate of mass degradation and deposition at time $t$ for the $j$ th constituent were expressed as

$$
\dot{\rho}_{t}^{j}=\rho_{t}^{j} k_{\sigma}^{j} \frac{\sigma_{t}^{j}-\sigma_{h}^{j}}{\sigma_{h}^{j}}+\dot{D}^{j}
$$

where $\rho_{t}^{j}$ is the mass density of the $j$ th constituent at time $t$ and $k_{\sigma}^{j}$ denotes a growth parameter while $\sigma_{t}^{j}$ and $\sigma_{h}^{j}\left(\sigma_{h}^{j}=\left(\boldsymbol{a}_{0}^{j} \otimes \boldsymbol{a}_{0}^{j}\right): \boldsymbol{\sigma}_{h}^{j}\right)$ are the current and homeostatic stresses, respectively. $\dot{D}^{j}$ includes any additional mass deposition or degradation governed by non-mechanical effects (for instance effect of a drug). A more general form of this equation is presented by Braeu et al [7], using a tensorial representation and possibly considering wall shear stress stimuli [20], 21]. The wall shear stress effects induced by the blood flow are neglected in our work here. Therefore, due to continuous mass deposition and removal, the traction-free configuration changed during G\&R, even if there is a balance between mass deposition and removal $\left(\rho_{t}^{j}=0\right)$. Mass deposition or removal occurred with a prestress which is different from the current stress at which mass is removed. Altogether this leads to changes of tissue microstructure referred as remodeling. Therefore, assuming that remodeling occurs at a constant volume, the evolution of the inelastic remodeling deformation gradient of the $j$ th constituent at time $t$ was expressed such as 2

$$
\left(\frac{\dot{\rho}_{t}^{j}}{\rho_{t}^{j}}+\frac{1}{T^{j}}\right)\left(\boldsymbol{S}_{t}^{j}-\boldsymbol{S}_{p r e}^{j}\right)=\left(2 \frac{\partial \boldsymbol{S}^{j}}{\partial \boldsymbol{C}_{e}^{j}}:\left(\boldsymbol{C}_{e}^{j} \boldsymbol{L}_{r}^{j}\right)\right) \text { with } \boldsymbol{L}_{r}^{j}=\dot{\boldsymbol{F}}_{r}^{j} \boldsymbol{F}_{r}^{j-1}
$$

where $\boldsymbol{S}^{j}$ is the second Piola-Kirchhoff while subscript "pre" indicates prestress. $\boldsymbol{L}_{r}^{j}$ denotes the remodeling velocity gradient and $T^{j}$ is the average turnover time during which old mass increment is degraded and replaced by a new mass increment. Prestress $\sigma_{p r e}^{i}$ is equal to the homeostatic stress $\sigma_{h}^{i}$, according to proposition 1 from Cyron and Humphrey [22]. On the other hand, the growth deformation gradient captures any local change of volume induced by 
mass variations of each constituent. Following Braeu et al [7], we assumed that all components of the mixture shared the same growth deformation gradient: $\boldsymbol{F}_{g}^{j}=\boldsymbol{F}_{g}$ obtained by summing the growth rate of each constituent: $\dot{\boldsymbol{G}}=\sum_{j=1}^{n} \dot{\boldsymbol{G}}^{j}$, where $n$ is number of constituents in the mixture. Following [7] the mass density in the current spatial configuration was related to the mass density in the reference configuration by

$$
\operatorname{det}\left(\boldsymbol{F}_{g}\right)=\frac{\rho_{t}}{\rho_{0}}
$$

Differentiating both sides and recalling that $J=\operatorname{det}(\boldsymbol{F})=\operatorname{det}\left(\boldsymbol{F}_{e}^{j}\right) \operatorname{det}\left(\boldsymbol{F}_{r}^{j}\right) \operatorname{det}\left(\boldsymbol{F}_{g}^{j}\right)=\operatorname{det}\left(\boldsymbol{F}_{g}^{j}\right)$, after some arrangement the rate of the growth deformation gradient for the $j$ th constituent was obtained by

$$
\dot{\boldsymbol{G}}^{j}=\frac{\dot{\rho}_{t}^{j}}{\rho_{t}\left(\boldsymbol{F}_{g}^{j}\right)^{-T}: \boldsymbol{B}^{j}} \boldsymbol{B}^{j}
$$

Note here that $\boldsymbol{G}^{j}$ is the growth rate tensor and it should not be confused with $\boldsymbol{G}_{h}^{j}$ which is the deposition stretch tensor.

Assuming anisotropic growth normal to the arterial wall and to fibers (thinning or thickening effects) and considering the through-thickness homogenization $\left(J=\lambda_{z} \lambda_{\theta} \lambda_{r}\right.$, with $\left.\lambda_{r}=\frac{h}{h_{0}}\right)$, the current thickness $(h)$ was derived as

$$
h=\frac{h_{0}}{\lambda_{\theta} \lambda_{z}} \frac{\rho_{t}}{\rho_{0}}
$$

where $h_{0}$ is the initial thickness, $\lambda_{\theta}$ and $\lambda_{z}$ are the circumferential and axial stretches, $\rho_{t}$ and $\rho_{0}$ are the time density and the initial density. Also we simplified the equations for remodeling 15 and growth 17 to the following expressions

$$
\begin{gathered}
\dot{\lambda}_{r}^{j}=\left(\frac{\dot{\rho}_{t}^{j}}{\rho_{t}^{j}}+\frac{1}{T^{j}}\right) \frac{\lambda^{j}}{\left(\lambda_{e}^{j}\right)^{2}} \frac{1}{\rho_{t} \phi^{j}}\left(\frac{\partial \Psi^{j}}{\partial \lambda_{e}^{j}}+\lambda_{e}^{j} \frac{\partial^{2} \Psi^{j}}{\left(\partial \lambda_{e}^{j}\right)^{2}}\right)^{-1} \times\left(\sigma_{t}^{j}-\sigma_{p r e}^{j}\right) \\
\dot{G}_{r}^{j}=\frac{\dot{\rho}_{t}^{j}}{\rho_{t}}
\end{gathered}
$$

where the remodeling stretch $\left(\lambda_{r}^{j}\right)$ was along the fiber direction $\left(\boldsymbol{a}_{0}^{j}\right)$, growth $\left(\dot{\boldsymbol{G}}^{j}\right)$ is along the thickness direction $\left(\boldsymbol{e}_{r}\right)$, and $\sigma^{j}$ is the Cauchy stress. Then, we wrote the following expressions for inelastic deformation gradients $\boldsymbol{F}_{r}^{j}$ and $\boldsymbol{F}_{g}^{j}$,

$$
\begin{gathered}
\boldsymbol{F}_{r}^{j}=\lambda_{r}^{j} \boldsymbol{a}_{0}^{j} \otimes \boldsymbol{a}_{0}^{j}+\frac{1}{\sqrt{\lambda_{r}^{j}}}\left(\boldsymbol{I}-\boldsymbol{a}_{0}^{j} \otimes \boldsymbol{a}_{0}^{j}\right) \\
\boldsymbol{F}_{g}^{j}=\boldsymbol{I}+\frac{\rho_{t}}{\rho_{0}} \boldsymbol{a}_{0}^{j \perp} \otimes \boldsymbol{a}_{0}^{j \perp}-\boldsymbol{a}_{0}^{j \perp} \otimes \boldsymbol{a}_{0}^{j \perp}
\end{gathered}
$$

where $\boldsymbol{I}$ is the identity second order tensor, $\boldsymbol{a}_{0}^{j}$ are vectors of the fiber directions and $\boldsymbol{a}_{0}^{j \perp}$ are the vectors normal to the fibers (thickness direction). Finally, the inelastic deformation 
gradient was derived from (21) and (22), yielding

$$
\boldsymbol{F}_{g r}^{j}=\lambda_{r}^{j} \boldsymbol{a}_{0}^{j} \otimes \boldsymbol{a}_{0}^{j}+\frac{1}{\sqrt{\lambda_{r}^{j}}}\left(\boldsymbol{I}-\boldsymbol{a}_{0}^{j} \otimes \boldsymbol{a}_{0}^{j}+\frac{\rho_{t}}{\rho_{0}} \boldsymbol{a}_{0}^{j \perp} \otimes \boldsymbol{a}_{0}^{j \perp}-\boldsymbol{a}_{0}^{j \perp} \otimes \boldsymbol{a}_{0}^{j \perp}\right) \Longrightarrow \boldsymbol{F}_{g r}^{j} \cdot \boldsymbol{a}_{0}^{j}=\lambda_{r}^{j} \boldsymbol{a}_{0}^{j}
$$

To calculate the G\&R deformation gradient over time we solved the system composed of Eqs. 14, 15 and 17 by performing explicit temporal integration. It was assumed that elastin cannot be produced during adulthood, it even undergoes a slow degradation with a half-life time of several decades. Therefore, elastin evolution was basically calculated based on its degradation rate. Moreover, it was assumed that SMCs do not experience any mass turnover, however they undergo remodeling due to collagen and elastin mass evolutions.

\subsection{Adaptation to axisymmetric shell formulation}

In the current work, we implemented the G\&R model described in the previous subsection in axisymmetric shell elements. This subsection introduces the axisymmetric shell element with an overview of its kinematics and of the principle of virtual work (for more details we suggest [23], [24]).

Consistently with the kinematics of axisymmetric shell elements, we assumed that coordinates of a material point in the reference configuraton, denoted $X$ in the shell space, can be expressed by the position of the shell mid-surface, $X_{M}$, and by their local thickness parametric coordinate $\xi$ as

$$
\boldsymbol{X}=\underbrace{\left[\begin{array}{c}
Z \\
R
\end{array}\right]}_{\boldsymbol{X}_{M}}+\xi \underbrace{\left[\begin{array}{c}
-\cos \theta \\
\sin \theta
\end{array}\right]}_{\boldsymbol{N}}
$$

where $N$ is a unit normal vector. Similarly for the position in the deformed configuration, denoted $\boldsymbol{x}$, it can be written

$$
\boldsymbol{x}=\underbrace{\left[\begin{array}{c}
Z+u \sin \theta-w \cos \theta \\
R+u \cos \theta+w \sin \theta
\end{array}\right]}_{\boldsymbol{x}_{M}}+\xi \underbrace{\left[\begin{array}{c}
\cos (\theta-\beta) \\
\sin (\theta-\beta)
\end{array}\right]}_{\boldsymbol{d}}
$$

with $\boldsymbol{x}_{M}$ being the position of the deformed shell mid-surface and $\boldsymbol{d}$ being a vector describing the rotation of the cross section with respect to the mid-surface. Assuming plane stress, the deformation gradient is reduced to

$$
\boldsymbol{F}=\left[\begin{array}{cc}
\lambda_{z} & 0 \\
0 & \lambda_{\theta}
\end{array}\right]
$$

With the aim to solve the mechanical equilibrium by the principle of virtual work, the work due to the intenal and external forces is developed here. The internal virtual work is split along the shell thickness to define different properties for the media and the adventitia layers (bi-layered arterial wall). Let $h_{0}$ and $h^{*}$ be the total wall thickness and the media thickness, respectively. $\xi$ being the radial position, inequalities $-h_{0} / 2 \leq \xi \leq h^{*}$ and $h^{*} \leq \xi \leq h_{0} / 2$ 
define the media and the adventitia layers, respectively. The radial integral was derived by applying the principle of virtual work such as

$$
\delta W_{i n t}(\boldsymbol{u}, \delta \boldsymbol{u})=2 \pi \int_{L}\left(\int_{-h_{0} / 2}^{h^{*}} \boldsymbol{S} \cdot \delta \boldsymbol{E} R d \xi+\int_{h^{*}}^{h_{0} / 2} \boldsymbol{S} \cdot \delta \boldsymbol{E} R d \xi+\epsilon h_{0} E_{z r} \delta E_{z r} R\right) d s
$$

Then it is linearized as

$$
\begin{aligned}
D \delta W_{i n t}(\boldsymbol{u}, \delta \boldsymbol{u}) \cdot \Delta \boldsymbol{u} & =2 \pi \int_{L}\left(\int_{-h_{0} / 2}^{h^{*}}(\delta \boldsymbol{E} \cdot \mathbb{C} \cdot \Delta \boldsymbol{E}+\boldsymbol{S} \cdot \Delta \delta \boldsymbol{E}) R d \xi\right. \\
& +\int_{h^{*}}^{h_{0} / 2}(\delta \boldsymbol{E} \cdot \mathbb{C} \cdot \Delta \boldsymbol{E}+\boldsymbol{S} \cdot \Delta \delta \boldsymbol{E}) R d \xi \\
& \left.+\epsilon h_{0}\left(\delta E_{z r} \Delta E_{z r}+E_{z r} \Delta \delta E_{z r}\right) R\right) d s
\end{aligned}
$$

where $\epsilon$ is a penalty related to the shear strain $E_{z r}$ constraining the rotation of the cross section 25.

The external virtual work comes from a follower load. In this case the load could be an internal pressure due to the blood in the artery or a force due to the stent pushing onto the artery.

$$
\delta W_{e x t}(\boldsymbol{u}, \delta \boldsymbol{u})=2 \pi \int_{-1}^{+1} \delta \boldsymbol{u} \cdot \hat{p}\left(-\boldsymbol{e}_{\theta} \times \boldsymbol{x}_{, \xi}\right) r d \eta
$$

Then it is linearized as

$$
D \delta W_{e x t}(\boldsymbol{u}, \delta \boldsymbol{u}) \cdot \Delta \boldsymbol{u}=2 \pi \int_{-1}^{+1} \delta \boldsymbol{u} \cdot\left\{\Delta \hat{p}\left(-\boldsymbol{e}_{\theta} \times \boldsymbol{x}_{,_{\xi}}\right) r-\hat{p}\left(\boldsymbol{e}_{\theta} \times \Delta \boldsymbol{u}, \xi\right) r-\hat{p}\left(\boldsymbol{e}_{\theta} \times \boldsymbol{x},_{\xi}\right) \Delta w\right\} d \eta
$$

where $\hat{p}$ is the load due to an internal pressure $\hat{p}=P_{i}$ or a stent (Fig. 2) $\hat{p}=C_{N} \delta$. $\Delta \hat{p}$ is the variation of the load along the element, $\Delta \hat{p}=0$ for blood pressure or $\Delta \hat{p}=-C_{N} \Delta w$ for the stent. In equation 30 we consider the term $\Delta \hat{p}$ for possible variations of the load, in order to incorporate the force of the stent onto the arterial wall.

Finally, the Newton's method is used to solve the mechanical equilibrium, yielding the following system

$$
\left(D \delta W_{\text {int }}-D \delta W_{\text {ext }}\right) \cdot \Delta \boldsymbol{u}+\left(\delta W_{\text {int }}-\delta W_{\text {ext }}\right)=0
$$

Following Voigt's notation [23] we reduced the order of stress, strain and elasticity tensors as below

$$
\boldsymbol{S}=\left[\begin{array}{c}
S_{z} \\
S_{\theta}
\end{array}\right], \boldsymbol{E}=\left[\begin{array}{c}
E_{z} \\
E_{\theta}
\end{array}\right], \mathbb{C}=\left[\begin{array}{cc}
C_{z} & C_{z \theta} \\
C_{\theta z} & C_{\theta}
\end{array}\right]
$$

\section{$2.4 \quad$ Finite element implementation}

The model was implemented in an in-house code for axisymmetric shell elements (section 2.3) using FORTRAN programming language along with implementation of the homogenized

CMT of G\&R. A simplified arterial model was defined as a shell cylinder (thin-walled) of 
length $L=100[\mathrm{~mm}]$, mean radius $R=10[\mathrm{~mm}]$ and wall thickness $h_{0}=1.41[\mathrm{~mm}]$. The cylinder was discretized with 2-node shell elements in 35 elements along the axial direction. A luminal reference pressure $P_{i}=100[\mathrm{~mm}]$ was assigned. In some cases we considered a stent into the artery with length $L_{s}=40[\mathrm{~mm}]$, as shown in Fig. 2. The long time term problem was solved by a forward Euler time integration scheme with a time step of 30 days. The spatial and time discretization was chosen based in a convergence study.

\section{$3 \quad$ Numerical experiments}

\subsection{Comparison with an existing G\&R model}

First we considered an axisymmetric single-layer cylindrical shell wall as previously studied by Cyron et al [2]. Using the algorithm presented by Mousavi and Avril [17], the reference configuration was defined in such a way to ensure that the circumferential component of elastin deposition stretch was mechanically in equilibrium with the reference pressure. Following the first example of Braeu et al [7], the arterial wall underwent elastin degradation varying temporally and spatially across the arterial wall with the following rate:

$$
\dot{D}^{e}(\boldsymbol{X}, t)=-\frac{\rho^{e}(\boldsymbol{X}, t)}{T^{e}}-\frac{D_{\max }}{t_{d a m}} \rho^{e}(\boldsymbol{X}, 0) e^{-0.5\left(\frac{z}{L_{d a m}}\right)^{2}-\frac{t}{t_{d a m}}}
$$

where $t_{d a m}$ and $L_{d a m}$ are the temporal and the spatial damage distribution parameters, respectively. $Z$ is the material position in the axial direction of the cylinder and $D_{\max }$ is the maximum damage. The first term in Eq. 31 refers to a normal elastin degradation by age while the second one is related to a sudden and abnormal local damage starting at $t=0$ with maximum value at the center of the cylinder $(Z=0)$ and fading at $Z=\frac{L}{2}$. The results obtained with the present model were compared with the corresponding results of Cyron et al [2] for different growth parameters, $k_{\sigma}^{c_{j}}$. Material parameters are listed in Table 2.

\subsection{Applications of the model}

After verification of the model on a single layer arterial wall, three different G\&R cases were considered for a bi-layered cylindrical artery:

1. a benchmark case was considered first with a regional and sharp degradation rate of elastin in the arterial wall to develop an aneurysm (case I).

2. a stent of length $L_{s}$ was deployed in the artery as shown in Fig. 2 and the induced G\&R undergone by the artery was predicted (case II).

3. after developing an aneurysm for several months due to elastin degradation (as in the first case), the implantation of a stent graft of length $L_{s}$ was modeled and the induced G\&R undergone by the artery was predicted (case III). 
The geometrical characteristics and boundary conditions of the different cases are shown in Fig. 2 and material parameters are summarized in Table 2 . For each case, we performed different sensitivity analyses on parameters such as the effect of the G\&R rate, the penalty in the shell element formulation, the media thickness, the stent over-sizing ratio (ratio between the radius of the stent at equilibrium stress-free conditions and the radius of the artery just before stent implantation) and the normal and tangential friction coefficient between the stent and the arterial wall. Those parameters are reported in Table 1 .

\begin{tabular}{lcc}
\hline Parameter & Values & Case \\
\hline Gain parameter & $\{0.01 ; 0.02 ; 0.03 ; 0.04\}$ & $(1)(3)$ \\
Over-sizing & $\{5 \% ; 10 \% ; 15 \% ; 20 \%\}$ & $(2)$ \\
Media thickness ratio & $\{0.15 ; 0.50 ; 0.85\}$ & $(1) 3$ \\
Shell penalty & $\left\{10^{5} ; 10^{7} ; 10^{9}\right\}$ & $(1)(3)$ \\
Normal Coefficient & $\{25 M P a ; 50 M P a ; 75 M P a\}$ & $(2)$ \\
\hline
\end{tabular}

Table 1: Sensitivity Analysis

\section{Results}

\subsection{Comparison with an existing G\&R model}

Aneurysm growth predicted by our model was compared against the corresponding results from Cyron et al [2] in Fig. 3. As expected, the single-layer cylindrical artery underwent excessive and unstable dilatation for small growth parameters while it recovered its stability after a transient period of growth for larger growth parameters. Elastin loss is responsible for altering the stress field, leading subsequently to the deposition of new collagen fibers. Therefore, stability (large $k_{\sigma}^{c}$ ) or instability ( $\operatorname{small} k_{\sigma}^{c}$ ) of the aneurysm growth is governed by the balance between elastin loss and new collagen production.

\subsection{Case I: benchmark case of a bi-layer arterial wall}

The degradation of elastin changed the stiffness at the center $(Z=0)$. From this imbalance, the G\&R model predicted an adaptation of the artery by collagen deposition, until reaching equilibrium again, 14. The turnover of collagen was directly proportional to the gain parameter $\left(k_{\sigma}^{c}\right)$, so for small gain parameters the arterial wall did not recover homeostasis (stability) and a bulge grew from this instabiblity as shown in Fig. 4 and 6 a. Herein the simulation with $k_{\sigma}^{c}=0.01 / T^{c}$ lead to a $18.2 \mathrm{~mm}$ radius whereas the simulation with $k_{\sigma}^{c}=0.04 / T^{c}$ lead to a $13.4 \mathrm{~mm}$ radius after 200 months of G\&R.

The turnover of collagen also depended on the previous amount of collagen as shown in equation 14. Then for arteries with less initial collagen, it was difficult to recover homeostasis. A similar effect was observed with arteries having a thick media, see for instance the case with 85\% media shown in Fig. 6 b. Penalties also had some effect (Fig. 6 c) with smaller radius reached for $\epsilon=1 \times 10^{9}$ (Fig. A.1 in supplemental materials). 


\begin{tabular}{|c|c|c|}
\hline \multicolumn{3}{|c|}{ Material parameters } \\
\hline$C^{e}$ & elastin neo-hookean parameter & $72[\mathrm{~J} / \mathrm{kg}]$ \\
\hline$C_{1}^{c_{i}}$ & Collagen: Fung exponential parameters & $1136[\mathrm{~J} / \mathrm{kg}]$ \\
\hline$C_{2}^{c_{i}}$ & & 11.2 \\
\hline$C_{1}^{m}$ & Smooth muscle: passive contribution & $15.2[\mathrm{~J} / \mathrm{kg}]$ \\
\hline$C_{2}^{m}$ & & 11.4 \\
\hline$S_{\text {actmax }}$ & Smooth muscle: active contribution & $54[k P a]$ \\
\hline$\lambda_{0}^{m}$ & & 0.8 \\
\hline$\lambda_{\max }^{m}$ & & 1.4 \\
\hline$\lambda_{a c t}$ & & 1.0 \\
\hline$\rho_{0}$ & Total initial density & $1050\left[\mathrm{~kg} / \mathrm{m}^{3}\right]$ \\
\hline \multicolumn{3}{|c|}{ Media fraction mass } \\
\hline$\phi_{M}^{e}$ & Elastin & $40 \%$ \\
\hline$\phi_{M}^{m}$ & Smooth muscle & $40 \%$ \\
\hline$\phi_{M}^{c_{1}}$ & Collagen: axial & $1 \%$ \\
\hline$\phi_{M}^{c_{4}}$ & Collagen: circumferential & $18 \%$ \\
\hline$\phi_{M}^{c_{2}}=\phi_{M}^{c_{3}}$ & Collagen: diagonal & $0.5 \%$ \\
\hline \multicolumn{3}{|c|}{ Adventitia fraction mass } \\
\hline$\phi_{A}^{e}$ & Elastin & $5 \%$ \\
\hline$\phi_{A}^{m}$ & Smooth muscle & $0 \%$ \\
\hline$\phi_{A}^{c_{1}}$ & Collagen: axial & $10 \%$ \\
\hline$\phi_{A}^{c_{4}}$ & Collagen: circumferential & $5 \%$ \\
\hline$\phi_{A}^{c_{2}}=\phi_{A}^{c_{3}}$ & Collagen: diagonal & $40 \%$ \\
\hline \multicolumn{3}{|c|}{ Deposition Stretch } \\
\hline$G_{h z}^{e}$ & Elastin: axial & 1.25 \\
\hline$G_{h \theta}^{e}$ & Elastin: circumferential & 1.34 \\
\hline$G_{h}^{m}$ & Smooth muscle & 1.1 \\
\hline$G_{h}^{c_{i}}$ & Collagen & 1.062 \\
\hline \multicolumn{3}{|c|}{ Growth and Remodeling parameters } \\
\hline $\begin{array}{l}T^{c_{i}}=T^{m} \\
T^{e}\end{array}$ & Collagen/Smooth muscle: turnover time & $101[$ days] \\
\hline$T^{e}$ & Elastin: mean life time & $101[$ years] \\
\hline
\end{tabular}

Table 2: Material parameters 


\subsection{Case II: adaptation after stent implantation}

In this case, the mechanobiological insult applied to the artery was the radial force of a stent. The stent also stretched the artery during its deployment as shown in Fig. 5 b, depending on the over-sizing ratio. But the stress decreased quickly during the first 30 months due to G\&R, until reaching nearly homeostasis. The time to recover homeostasis was shorter for $5 \%$ over-sizing than for $20 \%$ over-sizing, $k_{\sigma}^{c}=0.02 / T^{c}$ (Fig. 5 c).

However, homeostasis was never reached everywhere along the artery. A residual radius increase was systematically obtained at the edge of the stent. This dilatation was bigger for small gain parameters as shown in Fig. A.3 (supplemental material), for larger over-sizing ratios, for large stent stiffness (Fig. 7) and for thicker media (Fig. A.5).

\subsection{Case III: stent implantation after several years of aneurysm development G\&R}

In this case, the central radius obtained after several months of adaptation did not depend either on the gain parameters or on the media thickness (Fig. $8 \mathrm{a}$ and b). The stent over-sizing and the stent stiffness had a small but significant effect (Fig. 8 c and d).

However the major effects were on the stress obtained in the wall, which depended significantly on the gain parameters as shown in Fig. 9 b and c, Fig. A.10. After stent implantation, the artery had a fast adaptation (20 months) in the stent segment. As in case II, a residual radius increase developed at the edge of the stent, with significant effects of the gain and of the media thickness (Fig. A.10 and A.12).

\subsection{Computational details}

The simulations were performed in a Workstation Dell Precision 3620 (Intel Core i5-7500 3.40GHz, 16.4 GB RAM) with Linux OS. The code was compiled with GNU gfortran compiler on same machine. The time spent by simulation are shown in Table 3 .

\begin{tabular}{|l|cc|}
\hline & CPU Time $[\mathrm{s}]$ & Clock Time [s] \\
\hline Case I (200 G\&R steps) & 0.972 & 0.977 \\
Case I (180 G\&R steps) & 0.872 & 0.877 \\
Case II (180 G\&R steps) & 0.876 & 0.885 \\
Case III (80 G\&R steps and 100 G\&R steps) & 1.088 & 1.108 \\
\hline
\end{tabular}

Table 3: Computational costs time of every simulation.

\section{Discussion}

In this work, a new open-source FE model of vascular adaptation, with low computational cost, was introduced and applied to model aneurysm growth and stent implantation. We used a layer-specific shell model for the arterial wall. Although previous simulations used membrane 
models [2], [14], [16], [26], [27] and hexaedra elements [7], [8], shell models appear as very well adapted to modeling the deformations of the aorta and its G\&R behavior [14]. Moreover, the ratio between thickness and diameter, which has to be at least of 1 to 10 for a shell, evolves favorably as the aneurysm grows. On top of that, remodeling tends to naturally maintain a uniform stress field across the thickness.

A 2D axisymmetric membrane model has two degrees of freedom (DOF), while a shell model has at least three DOF, depending on the number of extra constraints [23], [24]. In our model, the third DOF is the bending angle and we added the shear strain as an extra constraint. Thanks to the latter, we used a penalty $(\epsilon)$ [25] to zero the through-thickness shear strain. Our simulations showed that high penalty coefficients, $\epsilon=1 \times 10^{9}$, can affect arterial adaptation and even induce instabilities in G\&R due to shear locking effects [23]. We found that the optimal penalty factor was $\epsilon=1 \times 10^{5}$, as it permitted a good compromise between shear strains, and displacements. However, after tuning the different parameters, differences remain between our results and the results from Cyron et al [2]. These differences may be attributed to the contribution of the bending behavior in our model, whereas Cyron et al [2] used membrane elements. The main argument for this interpretation is that the differences depend significantly on the penalty factor we use in our model to account for the through-thickness shear stiffness of the shell.

The simplification we have done in the fibre families enforce incompressiblity for elastic and remodeling deformation gradients and reduces the amount of unknowns to be solved at each time step. The assumption of anisotropic growth was shown to be more relevant to model adaptation after elastin degradation [7], [28]. Eriksson [28] even showed that inapropriate growth models could possibly induce counter-expected results such as arterial diameter shrinking.

An interesting feature of our model is the layer-specific implementation which allow to use different mass of constituents (elastin, collagen and SMC) in the mixture by layer and employ the G\&R separately. Indeed, there are mechanical differences between the media and the adventitia in the arterial wall due to the amount of constituents in each layer [29]. Then, it was assumed that homeostatic stresses are different in the media and in the adventitia, as they harbor different cell phenotypes (contractile smooth muscle cells in the media and fibroblasts in the adventitia). For instance, Bellini et al [30] also considered different stress distributions in the media and in the adventitia and showed that their model is well suited to predict the results of open angle experiments. The first bi-layered models of arterial wall in finite elasticity were proposed by Von Maltzahn et al [31] who reported discontinuous circumferential and axial stresses at the interface between the media and the adventitia. Different material parameters for the media and the adventitia permitted to reach nearly uniform, layer-specific circumferential stresses under physiologic conditions of pressure and axial stretch. Latorre and Humphrey 32 proposed a bi-layered model with a time-independent CMT approach. G\&R simulations with bi-layered models were performed using time-independent Rachev [33] and time-dependent Taber and Humphrey [34] approach. Both were based on the assumption that circumferential stress should be restored to their normal initial value. Taber and Humphrey [34] suggested that transmural differences in material properties were needed to predict the same opening angles as the ones observed experimentally. Finally this heterogeneity of material 
properties contributed to reach more uniform transmural distributions of stresses and to account for residual stresses. Herein, we proposed a bi-layered model for time-dependent G\&R. We defined layer-specific mass fractions of each constituent. Even if in both layers each constituent has the same strain energy density (per unit mass), each layer has a different strain energy density (per unit volume) due to the different mass fractions.

Following Wilson et al [35] and Braeu et al [7], elastin degradation was assumed to be the mechanobiological insult initiating G\&R simulations. Consistently with Wilson et al [16], it appeared that collagen production tends to compensate for the loss of elastin. Results demonstrated that the gain parameter, $k_{\sigma}$, has a key effect on collagen production and expansion rate, (Fig. 3) consistently with other studies [2], [26]. Nonetheless, the gain parameter was assumed to be fixed during the evolution of aneurysm in our model, whereas in an actual aneurysms gain may change during aneurysm growth due to pathological changes [27] affecting mechanosensing and mechanotransduction. The simulation showed that $k_{\sigma}=0.09$ provides almost a linear growth. With $k_{\sigma}<0.05$ the lesion enlarged continuously implying that the stress-mediated collagen turnover was not enough to return to the homeostatic stress. Another important factor in collagen production is the existing mass of collagen [16]. The initial collagen mass related to the initial thickness of adventitia, which has the larger mass fraction of collagen [36]. Simulations showed that only small dilatation were reached in 200 months (16.5 years) for $h_{M}=0.15 h_{0}$, whereas large lesions were obtained with $h_{M}=0.85 h_{0}$, due to the insufficient collagen turnover.

Aortic aneurysms can be repaired using stent-grafts [37], [38]. The stress distribution obtained after implanting a stent (we neglected the mechanical effect of the graft) in a bi-layer arterial wall has been studied extensively using the finite-element method [39]. However, the further adaptation had never been simulated using the CMT approach. Our results showed that G\&R induced an effect on the arterial wall similar to a stress relaxation effect at the long term [2]. This stress relaxation was dependent on the over-sizing ratio, see Fig. 5, with a possible instability occurring at the edge of the stent. The relaxation also affected the radius, see Fig. 9(c,f,i) and Fig. 5(c). Turnover (gain and existing mass) of collagen had no significant transient effects along the stent segment, see Fig. A.3 and Fig. A.5, but was responsible for the dilatation developed at the stent edge. We also showed in case III that the aneurysm diameter decreased after stent-graft implantation.

Furthermore, clinical evidence suggests the existence of issues after stenting, such as restenosis, stent thrombosis and arterial injuries. Kitahara et al [40] studied the impact of stent oversizing, concluding that aggressive oversizing may not lead to vascular injuries at the stent edge, as arteries with siginificant stent oversizing reach better apposition without increasing the amount of dissection at the stent egde. However, Chamie et al [41] concluded that the overstretching of the arterial wall due to an oversized stent was an important factor of dissection at stent edges. They also observed that the arterial wall responds to the injury through neointimal proliferation and vessel remodeling, then leading to restenosis around the stent. Finally, García-García [42] reported expansive vascular remodeling at the stent edge, which is also in agreement with our model prediction.

Finally, we emphasize that the model considered herein only simulates fusiform aneurysms as proposed by Baek et al [26] and Wilson et al [35]. Other limitations of this work are related 
to the contact between the artery and the stent, which is simply modeled by springs pushing the shell elements at its nodes. Additionally, intraluminal thrombus [27], [35], which often has an important role in the growth of aortic aneurysm, was neglected in our models. Further extension of our shell formulation to 3D is expected to address these limitations.

\section{Conclusions}

In this work, we implemented a low-cost open-source finite-element 2D axisymmetric shell model (FEM) of the arterial wall for simulating layer-specific growth and remodeling using the homogenized constrained mixture theory. After testing the reliability of the implementation, we used the model to evaluate the long-term mechanobiological adaptation after stent implantation. Two types of regime were found: either the artery recovered its initial homeostatic stress state after some months of adaptation (stable regime), or the artery dilated locally at the edge of the stent without recovering homeostasis (unstable regime). The main results are that the ratio between the media and the adventitia thicknesses and the gain parameters are the major parameters determining the type of adaptation regime undergone by the aorta after stent implantation. It will be essential to estimate their patient-specific values for real patientspecific applications.

Although our model was limited to axisymmetric examples with 2D shell elements, its main advantage was the computational time, which was extremely low, while still capturing the main aspects of G\&R. Further extension to 3D geometries of this shell formulation is expected to address more realistic cases.

\section{Conflict of interest}

There is no conflict of interest.

\section{Acknowledgements}

The authors are grateful to the European Research Council for grant ERC-2014-CoG BIOLOCHANICS.

\section{$9 \quad$ References}

[1] A. Cocciolone, J. Hawes, M. Staiculescu, E. Johnson, M. Murshed, and J. Wagenseil, "Elastin, arterial mechanics, and cardiovascular disease", Am J Physiol Heart Circ Physiol, vol. 315(2), H189-H205, 2018.

[2] C. Cyron, R. Aydin, and J. Humphrey, "A homogenized constrained mixture (and mechanical analog) model for growth and remodeling of soft tissue", Biomech Model Mechanobiol, vol. 15, pp. 1389-1403, 2016.

[3] J. Humphrey and K. Rajagopal, "A constrained mixture model for growth and remodeling of soft tissues", Math Models Methods Appl Sci, vol. 12(3), pp. 407-430, 2002. 
[4] E. Rodriguez, A. Hoger, and A. McCulloch, "Stress-dependent finite growth in soft elastic tissues", J Biomech, vol. 27, pp. 455-467, 1994.

[5] H. Hosseini, K. Garcia, and L. Taber, "A new hypothesis for foregut and heart tube formation based on differential growth and actomyosin contraction", Development, vol. 144(13), pp. 2381-91, 2017.

[6] H. Hosseini and L. Taber, "How mechanical forces shape the developing eye", Prog Biophys Mol Biol, pp. 1-12, 2018.

[7] F. Braeu, A. Seitz, R. Aydin, and C. Cyron, "Homogenized constrained mixture models for anisotropic volumetric growth and remodeling", Biomech Model Mechanobiol, vol. 16(3), pp. 889-906, 2017.

[8] W. Lin, M. Iafrati, R. Peattie, and L. Dorfmann, "Growth and remodeling with application to abdominal aortic aneurysms", J Eng Math, vol. 109(1), pp. 113-137, 2017.

[9] N. Famaey, J. Vastmans, H. Fehervary, L. Maes, E. Vanderveken, F. Rega, S. Mousavi, and S. Avril, "Numerical simulation of arterial remodeling in pulmonary autografts", $Z$ Angew Math Mech, pp. 1-19, 2018.

[10] A. Valentín and G. Holzapfel, "Constrained mixture models as tools for testing competing hypotheses in arterial biomechanics: A brief survey", Mech Res Commun, vol. 42, pp. 12633, 2012.

[11] A. Valentín, J. Humphrey, and G. Holzapfel, "A finite element-based constrained mixture implementation for arterial growth, remodeling, and adaptation: Theory and numerical verification", Int J Numer Method Biomed Eng, vol. 29(8), pp. 822-49, 2013.

[12] A. Valentín, J. Humphrey, and G. Holzapfel, "A multi-layered computational model of coupled elastin degradation, vasoactive dysfunction, and collagenous stiffening in aortic aging", Ann Biomed Eng, vol. 39(7), pp. 2027-45, 2011.

[13] P. Watton, N. Hill, and M. Heil, "A mathematical model for the growth of the abdominal aortic aneurysm", Biomech Model Mechanobiol, vol. 3(2), pp. 98-113, 2004.

[14] P. Watton and N. Hill, "Evolving mechanical properties of a model of abdominal aortic aneurysm", Biomech Model Mechanobiol, vol. 8(1), pp. 25-42, 2009.

[15] C. Cyron, J. Wilson, and J. Humphrey, "Mechanobiological stability: A new paradigm to understand the enlargement of aneurysms?", J. R. Soc. Interface, vol. 11, p. 20140680 , 2014.

[16] J. Wilson, S. Baek, and J. Humphrey, "Parametric study of effects of collagen turnover on the natural history of abdominal aortic aneurysms", Proc Math Phys Eng Sci, vol. 469(2150), p. $20120556,2013$.

[17] S. Mousavi and S. Avril, "Patient-specific stress analyses in the ascending thoracic aorta using a finite-element implementation of the constrained mixture theory", Biomech Model Mechanobiol, vol. 16(5), pp. 1765-1777, 2017. 
[18] S. Mousavi, S. Farzaneh, and S. Avril, "Computational predictions of damage propagation preceding dissection of ascending thoracic aortic aneurysms", Int J Numer Method Biomed Eng, vol. 34(4), e2944, 2018.

[19] T. Matsumoto and K. Hayashi, "Response of arterial wall to hypertension and residual stress", in Biomechanics, 1996, pp. 93-119.

[20] L. Taber, "A model for aortic growth based on fluid shear and fiber stresses", J Biomech Eng, vol. 120, no. 3, pp. 348-354, 1998.

[21] C. Figueroa, S. Baek, C. Taylor, and J. Humphrey, "A computational framework for fluid-solid-growth modeling in cardiovascular simulations", Comput. Methods Appl. Mech. Engrg., vol. 198, no. 45, pp. 3583 -3602, 2009.

[22] C. Cyron and J. Humphrey, "Vascular homeostasis and the concept of mechanobiological stability", Int J Eng Sci, vol. 52, pp. 645-664, 2016.

[23] P. Wriggers, Nonlinear Finite Element Methods. Springer Science \& Business Media, 2008.

[24] O. Zienkiewicz, R. Taylor, and D. Fox, The Finite Element Method for Solid and Structural Mechanics. Elsevier, 2014.

[25] W. Wagner, "A finite element model for non-linear shells of revolution with finite rotations", Int J Numer Methods Eng, vol. 29, pp. 1455-1471, 1990.

[26] S. Baek, K. Rajagopal, and J. Humphrey, "A theoretical model of enlarging intracranial fusiform aneurysms", J Biomech Eng, vol. 128(1), pp. 142-149, 2006.

[27] S. Zeinali-Davarani, A. Sheidaei, and S. Baek, "A finite element model of stress-mediated vascular adaptation: Application to abdominal aortic aneurysms", Comput Methods Biomech Biomed Eng, vol. 14, no. 9, pp. 803-817, 2011.

[28] T. Eriksson, P. Watton, X. Luo, and Y. Ventikos, "Modelling volumetric growth in a thick walled fibre reinforced artery", J Mech Phys Solids, vol. 73, pp. 134-150, 2014.

[29] A. Caulk, G. Tellides, and J. Humphrey, "Chapter 7 - vascular mechanobiology, immunobiology, and arterial growth and remodeling", in Mechanobiology in Health and Disease, S. Verbruggen, Ed., Academic Press, 2018, pp. 215-248.

[30] C. Bellini, J. Ferruzzi, S. Roccabianca, E. D. Martino, and J. Humphrey, "A microstructurally motivated model of arterial wall mechanics with mechanobiological implications", Ann Biomed Eng, vol. 42(3), pp. 488-502, 2014.

[31] W. V. Maltzahn, D. Besdo, and W. Wiemer, "Elastic properties of arteries: A nonlinear two-layer cylindrical model", J Biomech, vol. 14, no. 6, pp. 389-397, 1981.

[32] M. Latorre and J. Humphrey, "Critical roles of time-scales tissue growth and remodeling", APL Bioeng, vol. 2(2), p. 026 108, 2018.

[33] A. Rachev, "Theoretical study of the effect of stress-dependent remodeling on arterial geometry under hypertensive conditions", J Biomech, vol. 30, no. 8, pp. 819-827, 1997. 
[34] L. Taber and J. Humphrey, "Stress-modulated growth, residual stress, and vascular heterogeneity", J Biomech Eng, vol. 123, no. 6, pp. 528-535, 2001.

[35] J. Wilson, S. Baek, and J. Humphrey, "Importance of initial aortic properties on the evolving regional anisotropy, stiffness and wall thickness of human abdominal aortic aneurysms", J R Soc Interface, vol. 9, no. 74, pp. 2047-2058, 2012.

[36] A. Pérez-Martin, I. Aïchoun, and M. D. Rienzo-Ambrozkiewicz, "Chapitre 2 - rappels physiologiques: Organisation générale et régulation du système artériel et de l'endothélium", in Maladies Artérielles, Paris: Elsevier Masson, 2016, pp. 9-26.

[37] A. de Préville, D. Fabre, J. Sobocinski, R. Spear, A. Hertault, T. Martin-Gonzalez, R. Azzaoui, and S. Haulon, "Chapitre 27 - anévrismes de l'aorte thoracique", in Maladies Artérielles, Paris: Elsevier Masson, 2016, pp. 313 -317.

[38] E. Teo and E. Isselbacher, "34 - diseases of the aorta", in Essential Echocardiography, S. Solomon, J. Wu, and L. Gillam, Eds., Elsevier, 2019, 354 -368.e1.

[39] G. Holzapfel and T. Gasser, "Computational stress-deformation analysis of arterial walls including high-pressure response", Int J Cardiol, vol. 116, no. 1, pp. 78 -85, 2007.

[40] H. Kitahara, K. Okada, T. Kimura, P. Yock, A. Lansky, J. Popma, A. Yeung, P. Fitzgerald, and Y. Honda, "Impact of stent size selection on acute and long-term outcomes after drug-eluting stent implantation in de novo coronary lesions", Circ Cardiovasc Interv, vol. 10, no. 10, e004795, 2017.

[41] D. Chamié, H. Bezerra, G. Attizzani, H. Yamamoto, T. Kanaya, G. Stefano, Y. Fujino, E. Mehanna, W. Wang, A. Abdul-Aziz, M. Dias, D. Simon, and M. Costa, "Incidence, predictors, morphological characteristics, and clinical outcomes of stent edge dissections detected by optical coherence tomography", JACC: Cardiovasc Interv, vol. 6, no. 8, pp. $800-813,2013$.

[42] H. García-García, N. Gonzalo, S. Tanimoto, E. Meliga, P. de Jaegere, and P. Serruys, "Characterization of edge effects with paclitaxel-eluting stents using serial intravascular ultrasound radiofrequency data analysis: The betax (beside taxus) study", Rev Esp Cardiol, vol. 61, no. 10, pp. 1013-1019, 2008.

\section{List of Figures}

$1 \quad$ Schematics representing the kinematic variables of the shell axisymmetric element 22

2 Geometrical characteristics of the artery model with symmetric conditions at $Z=0$ and $Z=L . L, R, L_{s}$ and $\hat{p}$ denote the arterial length, arterial radius, stent length and the internal pressure, respectively. a: Schematic representation of a real stent deployed in an artery with its media and adventitia, and the square shows the domain over which the simulations are performed (assuming symmetries). b: Simple geometrical model and its boundary conditions in a cylindrical coordinate system. . . . . . . . . . . . . . . . . . 23 
3 Changes of the radius in the center of arterial wall with maximum elastin degradation rate $(Z=0)$. Solid lines are the results obtained by the shell model and dashed lines are the results of $[2] . \ldots \ldots \ldots \ldots$. . . . . . . . . . . . . 24

$4 \quad$ Effects of gain parameters on the aneurysm growth after elastin loss. a, b and c are plotted in the center of the arterial wall $(Z=0)$ versus time. $\mathrm{d}$, e and f are plotted for the last time step (after 199 months) along the arterial axial

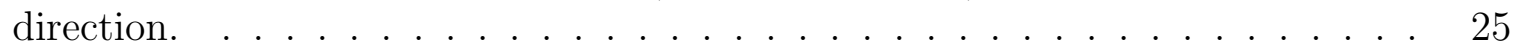

5 Effect of the stent over-sizing on G\&R of the arterial wall. a, b and c are plotted in the center of the arterial wall $(Z=0)$ versus time. $\mathrm{d}$, e and $\mathrm{f}$ are plotted for the last time step (after 180 months) along the arterial axial direction. . . . . . 26

6 Sensitivity analysis on the radius at $Z=0$ after 200 months of elastin degradation. a: Effect of different gain parameters. b: Effect of the media thickness. c: Effect of the penalty. . . . . . . . . . . . . . . . . . 27

$7 \quad$ Sensitivity analysis on the at $Z=0$ after 180 months following stent implantation. a: Effect of different gain parameters. b: Effect of the media thickness. c: Effect of stent over-sizing. d: Effect of the stent stiffness. . . . . . . . . . . 28

$8 \quad$ Sensitivity analysis on the radius at $Z=0$ after 100 months following stent implantation preceded by 80 months of aneurysm growth. a: Effect of different gain parameters. b: Effect of the media thickness. c: Effect of stent over-sizing. d: Effect of the stent stiffness. . . . . . . . . . . . . . . . . . . . . . 29

$9 \quad$ Effect of gain parameters and stent over-sizing on the behavior of the arterial wall after stent implantation for an arterial wall undergoing several years of G\&R. a, b and c are plotted at the center of the arterial wall $(Z=0)$ versus time. $\mathrm{d}$, e and $\mathrm{f}$ are plotted just before stent implantation $(\mathrm{t}=80$ months) along the arterial axial direction. $\mathrm{g}, \mathrm{h}$ and $\mathrm{i}$ are plotted just after stent implantation $(\mathrm{t}=80$ months) along the arterial axial direction. $\mathrm{j}, \mathrm{k}$ and $\mathrm{l}$ are plotted after 100 months following stent implantation along the arterial axial direction. . . . . . 30 


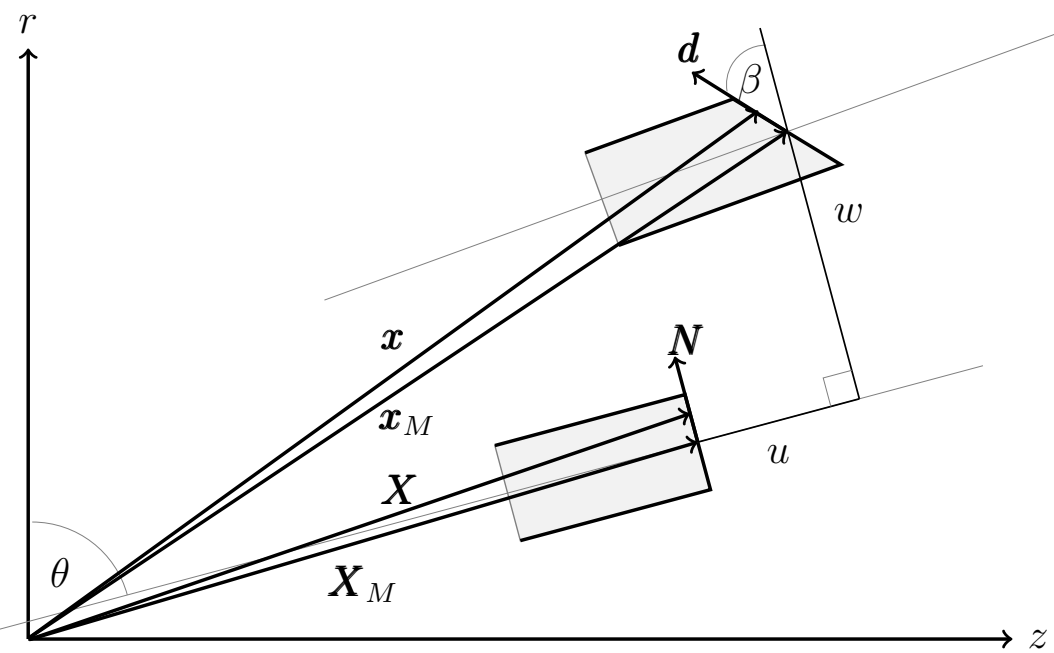

Figure 1 


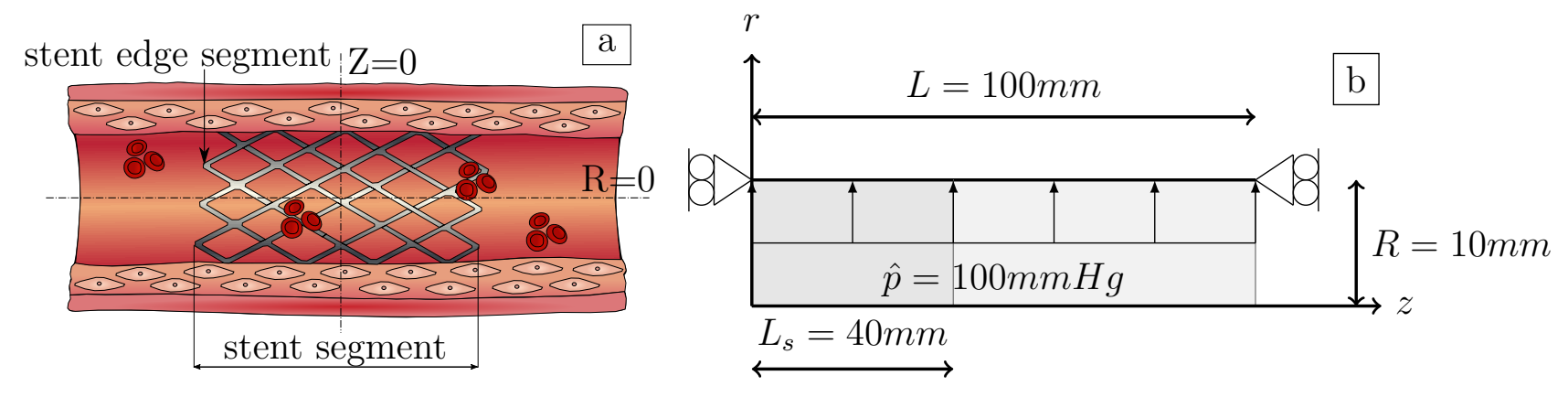

Figure 2 


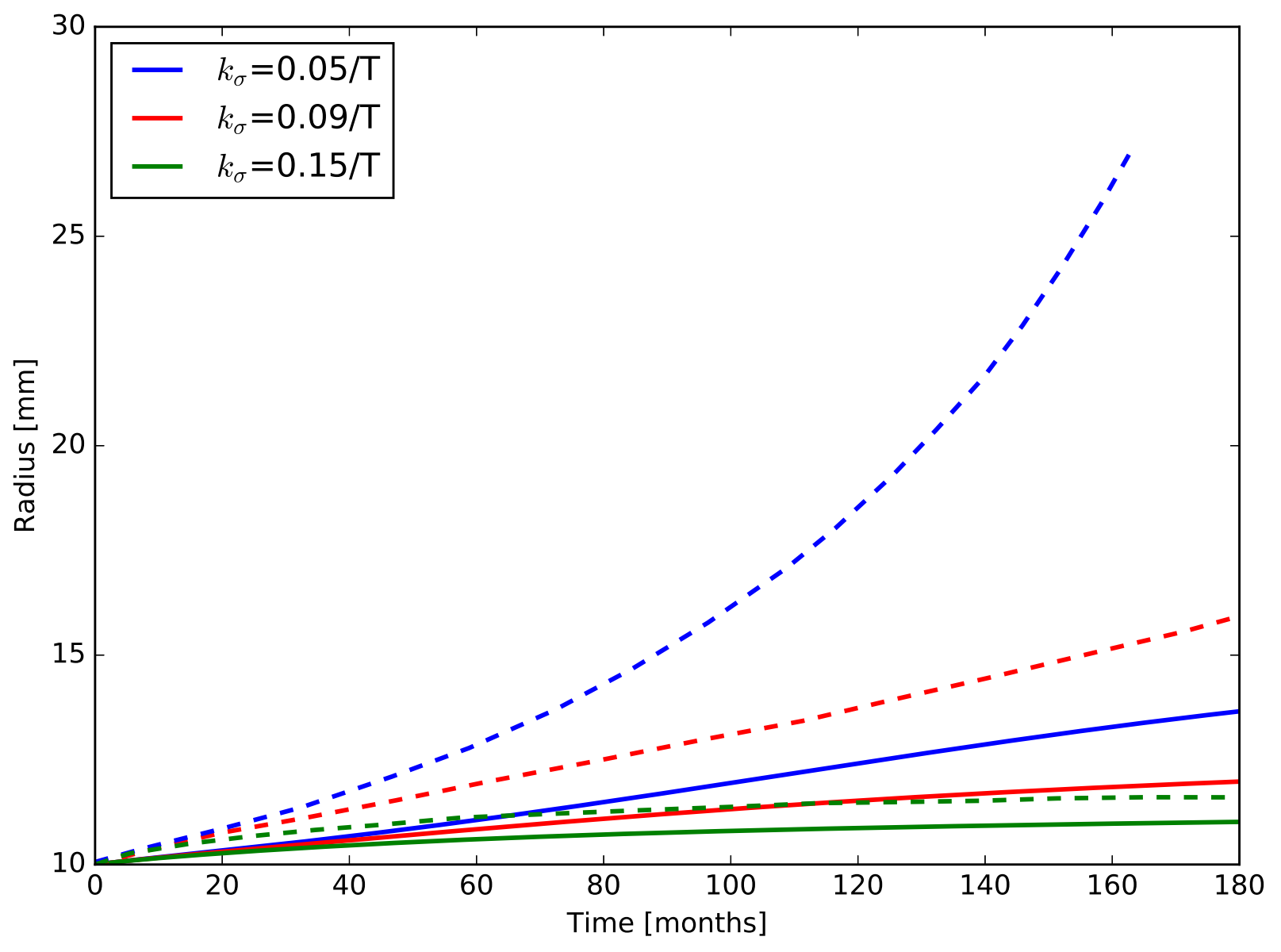

Figure 3 

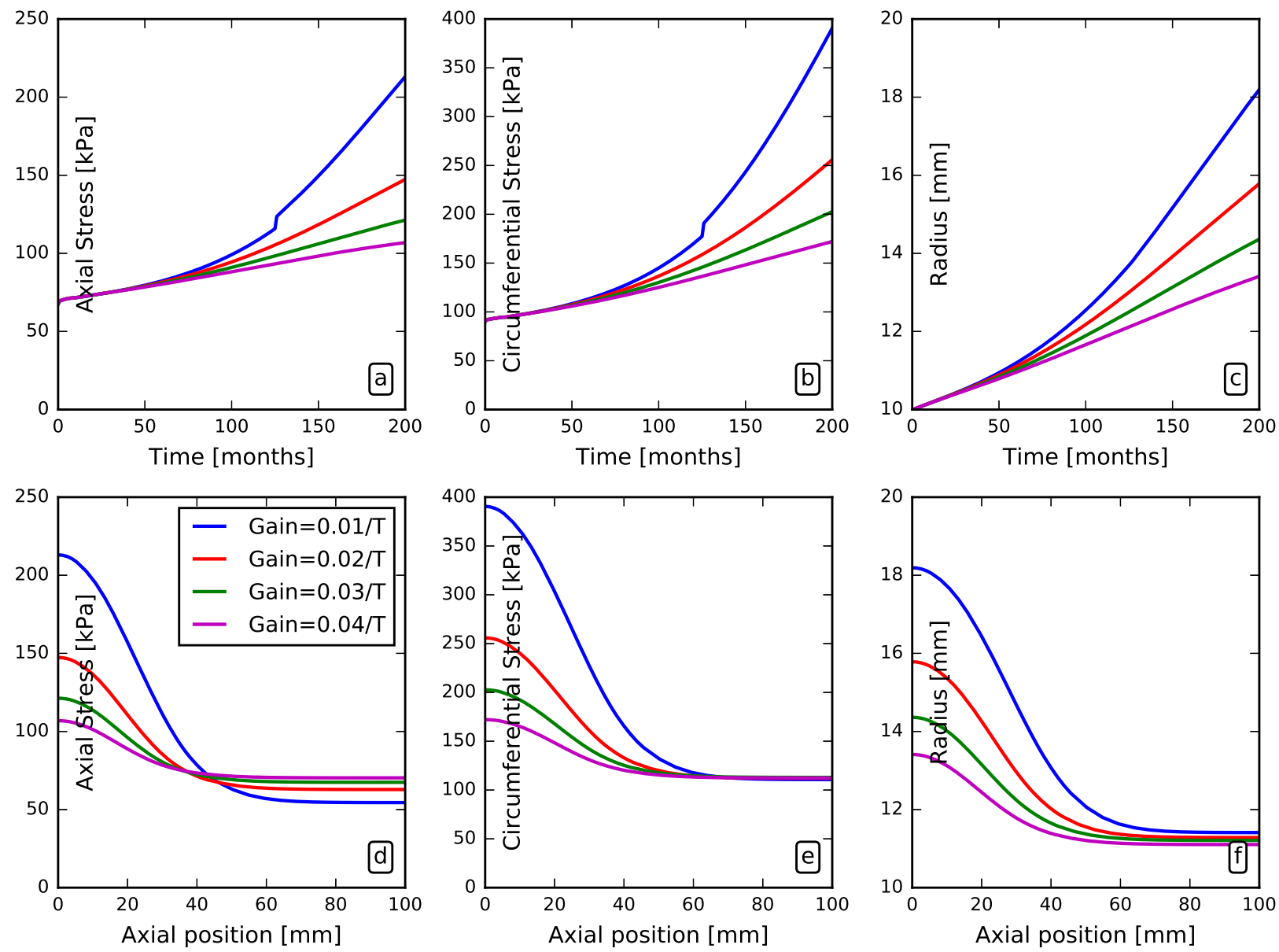

Figure 4 

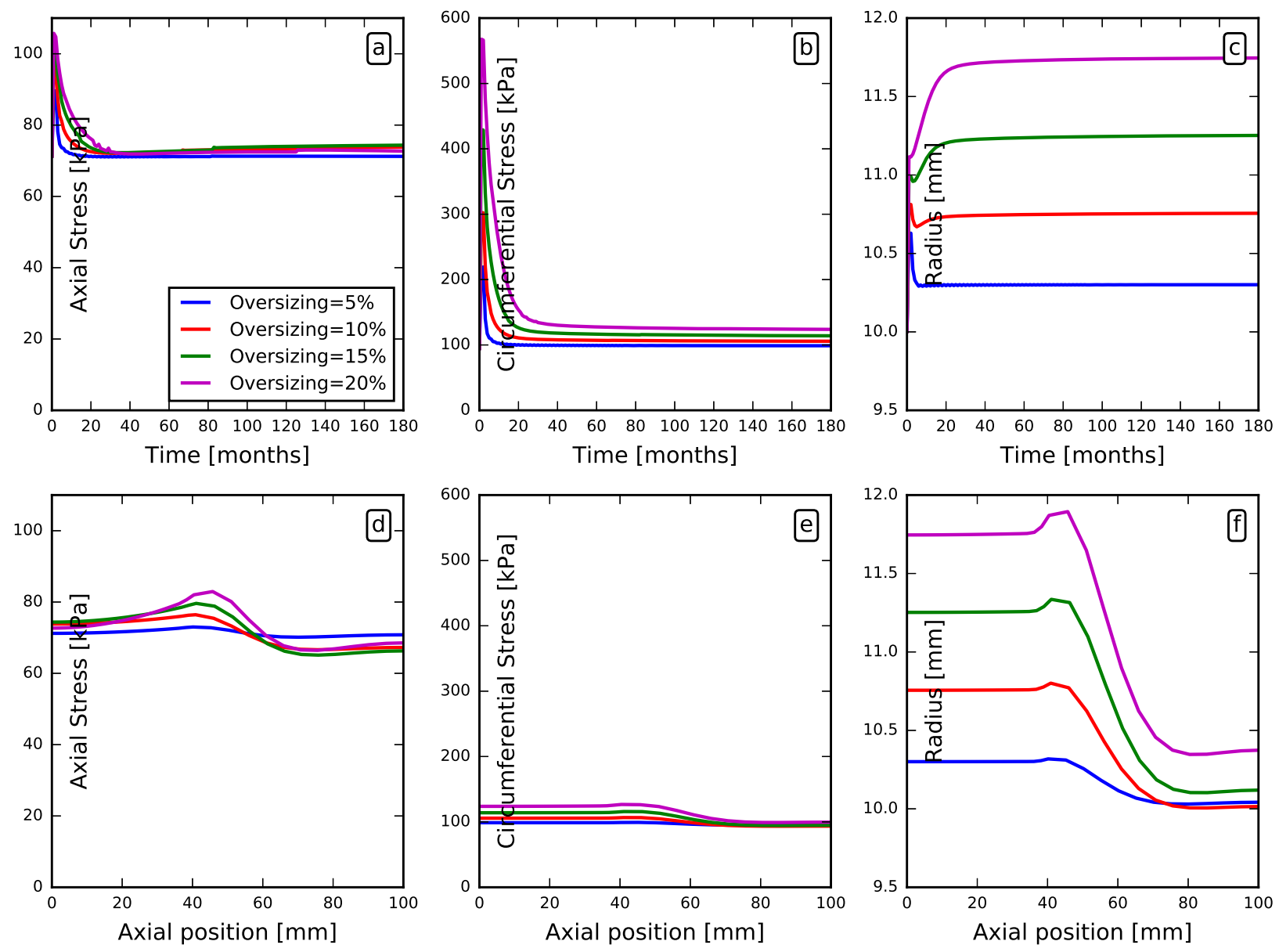

Figure 5 

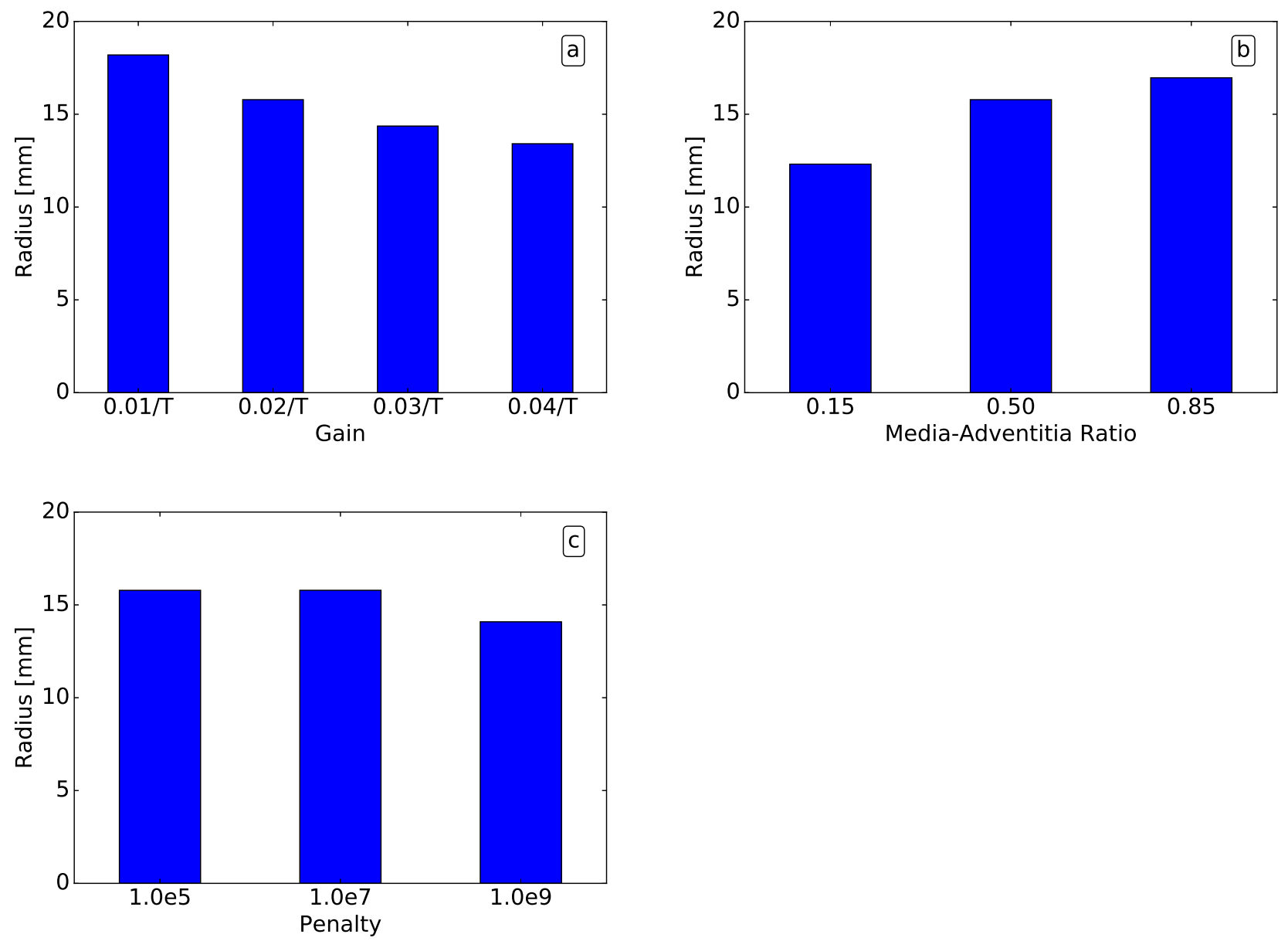

Figure 6 

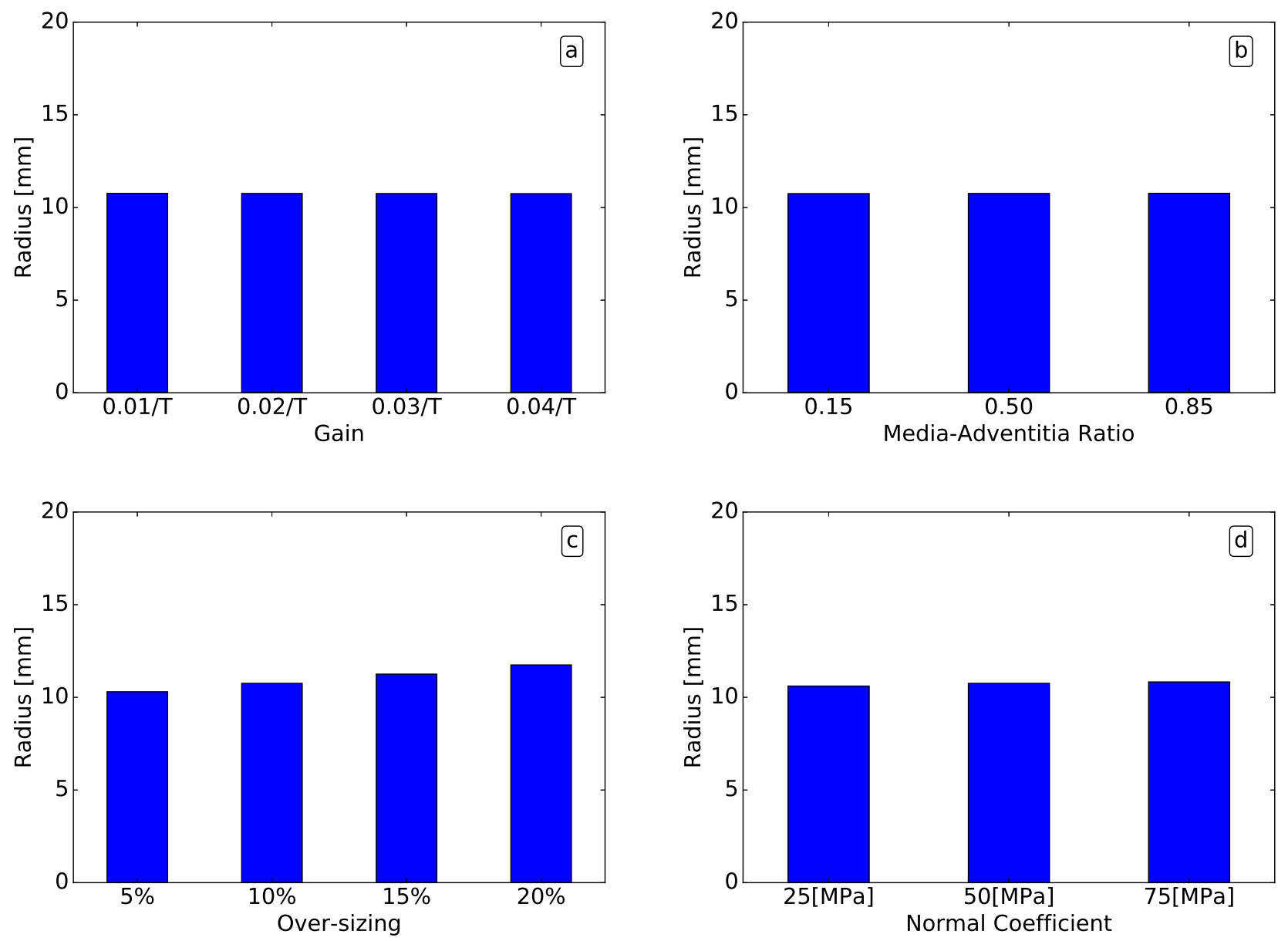

Figure 7 

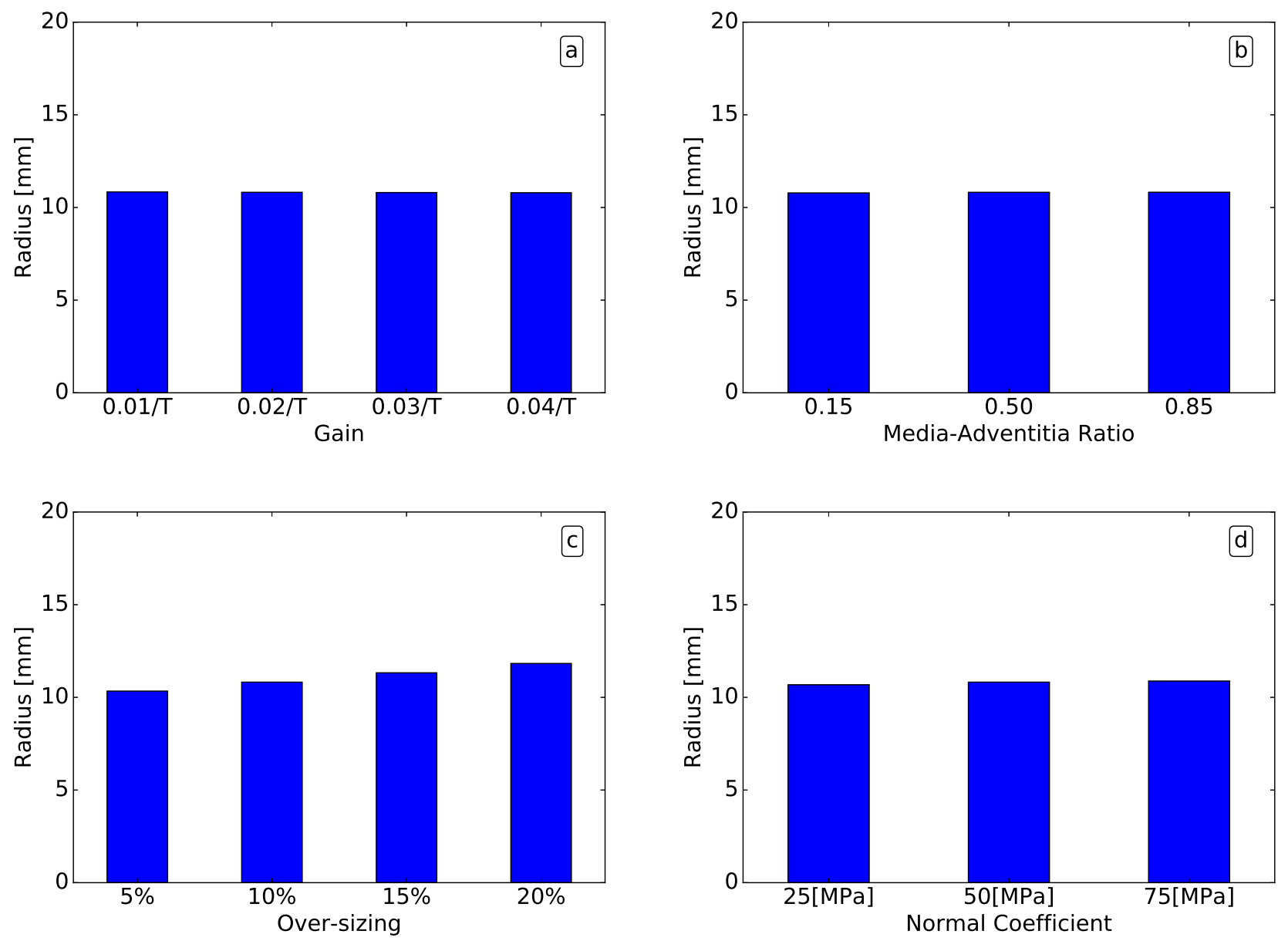

Figure 8 

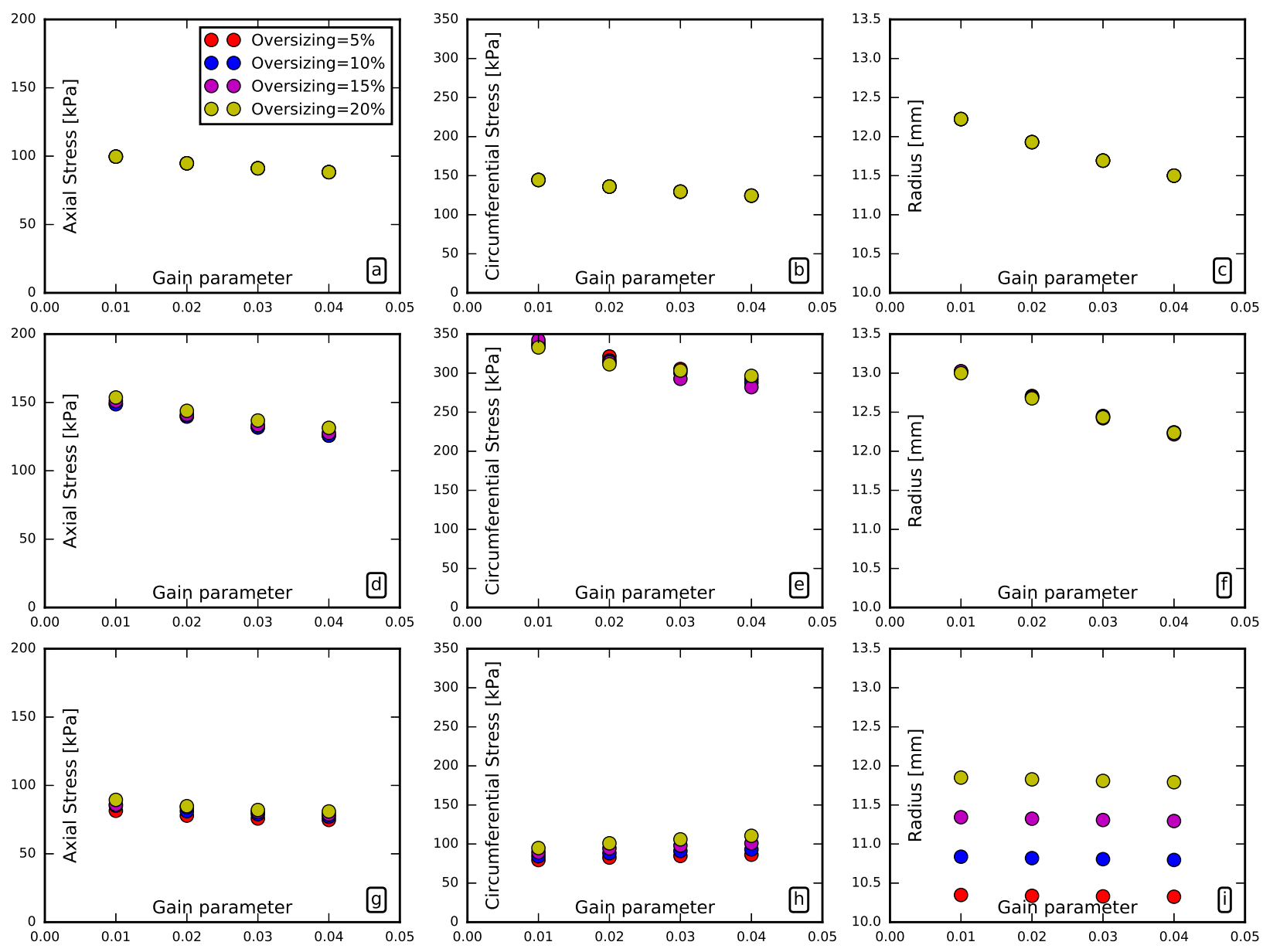

Figure 9 